\title{
Depressive Symptom Screening in Youth - Using a Community Diabetes Prevention Clinic
}

\author{
Alexander Wright ${ }^{1}$, Lisa Yazel-Smith ${ }^{2,3}$, Tamara Hannon ${ }^{2,3}$ \\ ${ }^{1}$ Indiana University School of Medicine, ${ }^{2}$ Department of Pediatrics, Indiana University School of \\ Medicine, ${ }^{3}$ Department of Pediatric and Adolescent Comparative Effectiveness Research, \\ Indiana University School of Medicine
}

Background: Rates of youth diagnosed with diabetes and prediabetes are increasing and comorbidities like depression warrant strict monitoring to improve patient outcomes.

Objective: The aim was to determine if increased depressive symptoms are associated with worse clinical findings, including BMI, obesity, diabetes, prediabetes, hemoglobin A1c (HbA1c), and demographic variables including age, gender, and race.

Methods: The study is a retrospective chart review of patients referred to the Youth Diabetes Prevention Clinic (YDPC) based on increased risk for type 2 diabetes (T2D). The Patient Health Questionnaire (PHQ-9), a 9-question depressive symptom screening tool, was utilized to screen for depressive symptoms using a cut point of $>=10$ which indicates possible depression and a need for further evaluation. All available data were evaluated for clinic visits occurring between 2013 and 2020.

Results: 178 patients filled out PHQ-9 screening forms and returned them to clinic. Age ( $p$ $<0.01)$, sex $(p<0.01)$, BMI $(p<0.01), \operatorname{HbA} 1 \mathrm{c}(p<0.01)$, and obesity diagnosis $(p=0.01)$ were associated with increased depressive symptoms based on screening score cutoff. Race $(p>$ $0.05)$, BMI z-score $(p=0.18)$, prediabetes diagnosis $(p=0.92)$, and diabetes diagnoses $(p=$ 0.05 ) were not. Middle adolescent (ages 14-17) youth were 3.5 times more likely than early adolescents to fall into the moderate/high risk category for depression. Females were 2.6 times more likely and those with higher $\mathrm{HbA} 1 \mathrm{c}$ or an obesity diagnosis were $\sim 3$ times more likely. A modest portion (36.5\%) in this sample met criteria for possible clinical depression.

Conclusion and Impact: Patients at risk for prediabetes and diabetes should be screened for depressive symptoms in order to intervene to decrease its adverse effects on diabetes management. Older youth, females, and those with obesity and higher $\mathrm{HbA1c}$ values are at particularly higher risk of having increased depressive symptoms. 